http://jmscr.igmpublication.org/home/ ISSN (e)-2347-176x ISSN (p) 2455-0450

crossref DOI: https://dx.doi.org/10.18535/jmscr/v8i2.123

Journal Of Medical Science And Clinical Research

\title{
A comparative study of self-evaluation questionnaire (STAI score) and Serum cortisol levels with relation to academic score in male and female first year MBBS students at the time of examination stress
}

\author{
Authors \\ Niraj Srivastava ${ }^{1}$, Geeta Batra Srivastava ${ }^{2}$ \\ ${ }^{1}$ Professor, Department of Physiology, Dr B S A Medical College \& Hospital, Rohini, Delhi, India \\ ${ }^{2}$ Consultant Pediatrician, Child Care Centre, Ghandhi Nagar, Ghaziabad \\ *Corresponding Author \\ Niraj Srivastava \\ Department of Physiology, Dr B S A Medical College \& Hospital Rohini, Delhi, India
}

\begin{abstract}
The current study was conducted in the Department of Physiology, Pt JLN Govt. Medical College \& Hospital, Chamba, HP on first year MBBS students.

The purpose of the study was to determine the effect of examination stress measured by self-evaluation questionnaire (STAI score), correlation of examination stress with Serum cortisol level and impact of examination stress on the academic score. The evaluation was done on three different intervals i.e., Pre examination, during Examination and Post examination (two weeks after the examination).

Two parameters were used to measure the level of stress; Spielberger State Trait Anxiety Inventory (STAI score) self evaluation questionnaire and serum cortisol level.

The study revealed that the group with elevated anxiety (score>40) was associated with significantly increased serum cortisol levels. The anxiety levels were decreased after examination period.

In most of the students expected pattern of increased anxiety during examination and decreased anxiety scores in post- examination were observed. As compared to male students, female students showed increased reactivity of cortisol during examination, which supported the evidence of increased stress response in females. The student's performance was affected by increased stress level measured by the parameters mentioned.

The present study adds to the literature of the level of stress during examination and its impact on performance in MBBS first year students.

Keywords: Examination Stress, Serum Cortisol, Anxiety, Effect on Performance, STAI.
\end{abstract}

\section{Introduction}

The stress system is essential for individual and survival of species. In a medical term stress is a physical, mental, or emotional factor that causes tension. The normal stress system function is important for maintenance of mental as well as physical health. While derangement of stress system causes patho-physiology. Indeed stress fully invade our life and influences us as individuals, communities, and humanity. Our body reacts to stress by activating a complex reservoir of behavioral and physiologic responses. 
There is general agreement that early adulthood is a challenging period of life, during which significant physical as well as psychological changes take place.

Aim of present education in medical institution is graduating knowledgeable, skillful and mentally healthy physicians that will able to handle their patients' with empathy. However, studies show that medical doctors experience high levels of stress related anxiety ${ }^{10}$. There is increasing awareness of stresses involved in medical training. ${ }^{1,2}$ Particularly examinations are a major cause of stress. In certain individuals stress may be beneficial in neurocognitive domain and task performance. High level of stress may have adverse physiological as well as psychological effect seen as decline in academic achievement.

Normally the physiological response of stress is characterized by stimulation of nervous system and endocrine system. The most important endocrine response is release of cortisol. But there are individual variation in its release. High versus low cortisol responders may represent two different groups. However not much information is available regarding the interrelationship of stress and academic performance in medical students.

The present study adds to the literature of the level of stress during examination and its impact on performance in MBBS first year students.

\section{Objective of the present Study}

- To determine the effect of examination stress by self-evaluation questionnaire (STAI score).

- To correlate examination stress with cortisol level

- To observe the impact of examination stress on the academic performance of first year medical students

- To compare level of cortisol in male and female medical students at the time of stress

\section{Materials and Methods \\ Study Design}

A prospective study was conducted on first year medical students to determine the examination stress measured by anxiety questionnaire (STAI score) and level of serum cortisol.

\section{Subjects}

A total of fifty first year MBBS students, age group 18- 22 years females and males were included in study.

\section{Inclusion criteria}

All healthy first year medical students who were mentally and physically fit were included.

\section{Exclusion criteria}

Students with any illness, fever or on drug treatment, smokers were excluded from the study.

\section{Setting of study}

The study was conducted in the department of Physiology, Pt JLN Govt. Medical College \& Hospital Chamba, H.P. for academic year 201920.

The evaluation was done at three intervals:

-Pre examination

- During the first terminal examination

-Post examination (two weeks after the first terminal examination)

The subject participation was purely voluntary basis and written informed consent was obtained before beginning of the study.

Two parameters were used to assess the level of stress

- Spielberger State Trait Anxiety Inventory (STAI) self-evaluation questionnaire

- $\quad$ Serum cortisol level

\section{STAI self evaluation questionnaire}

To measure the level of stress, Spielberger State Trait Anxiety Inventory (STAI) self-evaluation questionnaire was implemented. The STAI inventory consists of two scales:

First, the State Anxiety Scale (S- Anxiety) which evaluates the current state of anxiety or anxiety linked with some event by asking how subjects feels at present by using terms that measure subjective feelings like apprehension, 
nervousness, worry, and tension etc.

Second, the Trait Anxiety Scale (T-Anxiety) evaluates the personal characteristic of the subject. Each section of test consists of 20 items.

\section{Method of implementation of test}

Test is performed by using paper and pencil. This is a self- report based questionnaire that can be implemented in an individual format. Proper instructions are given for each of the S-Anxiety and T- Anxiety subscales.

The students have no time limit for responding to the questionnaire. However 10-15 minutes are allotted to complete both sets.

\section{Scoring}

Students respond to each STAI item by rating themselves on a four point scale. Range of scores for each subtest is 20-80, the higher score indicating greater anxiety.

The students were subdivided into two groups based on STAI state scores with those scoring $\leq$ 40 considered as low anxiety group while those with a score of $>40$ were considered as high anxiety group.

\section{Serum cortisol}

Early morning sample of $3 \mathrm{ml}$ of venous blood was collected in Vacutainer blood collection tube and sent immediately to biochemistry laboratory.
Analysis of Data

Mean \pm SD were calculated for the parameters analyzed and were compared by Student's t test by using SPSS 16 software.

P-values considered significant were as follows:-

$P<0.05-$ As significant

$P<0.001-$ As highly significant

\section{Result}

The evaluation was done at three different intervals which is as follows:

1) Pre examination

2) During the first terminal examination

3) Post examination (two weeks after the first terminal examination)

The pre- examination recordings were taken as baseline values.

The study participants included 30 boys and 20 girls. The data for the group was expressed as mean \pm standard deviation (SD). Means were compared between groups at three different times and within the groups, and correlated with each parameters and performance during the examination.

\section{Comparison of State and Trait Anxiety Score (STAI)}

State scale (S-Anxiety) was sensitive indicator of changes in levels of anxiety. Comparison of scores were done using Student's t test.

Table - 1: Comparison of STAI state (S) and trait $(\mathrm{T})$ anxiety scores on three intervals

\begin{tabular}{|l|c|c|c|}
\hline STAI & Pre-examination & Examination & Post-examination \\
\hline State $(\mathbf{N}=\mathbf{5 0})$ & $40.13 \pm 10.20$ & $45.10 \pm 12.10$ & $37.59 \pm 8.76^{*}$ \\
\hline Trait $(\mathbf{N}=50)$ & $42.84 \pm 10.10$ & $45 \pm 10$ & $40.2 \pm 8.50$ \\
\hline Statistically Significant
\end{tabular}

STAI scores increased from baseline level to examination. (Table1) examination and decreased in post-examination period. Statistically significant decreased $(\mathrm{P}=<0.05)$ was observed in state anxiety ( $\mathrm{S}$ Anxiety) scores from examination to post-

\section{Comparison of Cortisol Levels}

Elevated serum cortisol levels from baseline were observed during Examination period.

Table -2: Comparison of Serum Cortisol levels on two intervals

\begin{tabular}{|l|c|c|}
\hline Serum cortisol & Pre-examination & Examination \\
\hline Cortisol $(\mathbf{n g} / \mathbf{m l})(\mathbf{N}=\mathbf{5 0})$ & $60.48 \pm 17.70$ & $145.11 \pm 62.95^{*}$ \\
\hline
\end{tabular}
Thtatistically significantly
The rise in Serum cortisol was significant $(\mathrm{P}=<0.05)$. (Table 2$)$


Comparison of STAI Anxiety Scores in Boys and Girls on Three Intervals

Table - 3: Comparison of STAI score in boys and girls on three different intervals

\begin{tabular}{|l|c|c|c|}
\hline & Pre-examination & Examination & Post-examination \\
\hline Boys (N=30) & $40.30 \pm 11.44$ & $44.70 \pm 9.57$ & $39.60 \pm 9.78$ \\
\hline Girls (N=20) & $35.33 \pm 12.08$ & $45.77 \pm 14.99$ & $36.25 \pm 5.36^{*}$ \\
\hline
\end{tabular}

*Statistically significant $(\mathrm{P}=<0.05)$

Both boys and girls showed the same pattern of increased anxiety scores from baseline to examinations and decreased STAI scores during post- examination. However in girls decreased STAI scores during post-examination were statistically significant $(\mathrm{P}=<0.05)$. (Table 3$)$

\section{Comparison of Serum Cortisol Levels in Boys and Girls on Three Intervals}

Table -4: Serum Cortisol levels in boys and girls on two different occasions

\begin{tabular}{|c|c|c|}
\hline & Pre-examination & Examination \\
\hline Boys $(\mathbf{N}=\mathbf{3 0})$ & $53.50 \pm 14.70$ & $119.7 \pm 31.10^{*}$ \\
\hline Girls $(\mathbf{N}=\mathbf{2 0})$ & $59.41 \pm 21.60$ & $211.10 \pm 78.50 *$ \\
\hline Statistically significant $(\mathrm{P}=<0.05)$
\end{tabular}

It was observed that baseline Serum cortisol levels were more in girls. Boys as well as girls showed significant rise of Serum cortisol $(\mathrm{P}<0.05)$ from baseline to examination. The rise of Serum cortisol in girls were statistically significant $(\mathrm{P}$ $<0.05)$ compared to boys during examinations. (Table 4).

\section{Comparison of STAI and Cortisol on Three Intervals}

Table-5: Comparisons of Serum cortisol and examination score in the students classified in low (STAI $\leq$ 40) and high anxiety (STAI> 40) at baseline on three different intervals.

\begin{tabular}{|c|c|c|c|c|}
\hline \multirow[b]{2}{*}{$\begin{array}{l}\text { STAI } \\
\text { Subgroups }\end{array}$} & \multicolumn{3}{|c|}{ Cortisol } & \multirow[b]{2}{*}{ Examination Score } \\
\hline & Pre- examination & Examination & $\begin{array}{c}\text { Post- } \\
\text { examination }\end{array}$ & \\
\hline$\leq 40(N=36)$ & $51.12+19.78$ & $153.43+78.77$ & - & $56.61+4.76$ \\
\hline$>40(N=14)$ & $62.15 \pm 11.41 *$ & $134.3 \pm 38.23$ & - & $49.53 \pm 6.25 * *$ \\
\hline
\end{tabular}

$*$ Statistically significant $(\mathrm{P}=<0.05)$ increase in cortisol level in high anxiety group.

** Statistically significant $(\mathrm{P}=<0.05)$ low examination score.

STAI - subgroups showed linear correlation with cortisol levels when observed at baseline; high anxiety ( $>40)$ group showed significantly elevated $(\mathrm{P}=<0.05)$ cortisol compared to low anxiety $(\leq 40)$ group. These students classified under low and high anxiety group at baseline when followed during examination showed decreased cortisol levels in high anxiety group. (Table 5)

At baseline readings students with low anxiety with low cortisol levels had statistically significant $(\mathrm{P}<0.05)$ better performance compared high anxiety group containing higher cortisol levels. (Table 5)

\section{Discussion}

The current study was conducted in the department of Physiology, Pt JLN Govt Medical College, Chamba,HP on first year MBBS students on three different intervals ie., Pre examination, during Examination and Post examination (two weeks after the completion of examination) to determine the effects of examination stress on academic performance.

To measure the level of stress, STAI anxiety scale was used and is considered to be a valid instrument. To assess the biochemical change in stress, estimation of Serum cortisol was chosen as it is the potential biomarker for stress.

In this study it was noticed that anxiety scores 
were elevated from baseline to examination and significantly decreased during post examination period. These findings were consistent with reports of C.B. Arndt. ${ }^{3}$

The current study showed statistically significant increase $(p<0.05)$ in cortisol levels from baseline to examinations. These findings were similar to previous studies that describe stress in the form of continuous mental tasks. ${ }^{4,5}$

In the present study it was observed that students facing examination stress with increasing serum cortisol showed better performance.

It was observed that higher anxiety scores (STAI $>40)$ were associated with higher cortisol levels when baseline readings were considered. This is supported by many earlier studies which found elevated serum cortisol levels in the group with high anxiety scores. ${ }^{6,7}$

Both boys and girls showed a significant rise $(p<0.05)$ in Serum cortisol from baseline to examinations and girls have higher levels of cortisol as compared to boys at baseline recording suggesting that estradiol played a significant role at the time of stress. ${ }^{8}$

In the present study it was also observed that high anxiety decreased the performance (scoring). This type of negative association between anxiety and performance was also observed by the previous studies. $^{9}$

\section{Conclusion}

As stress during examinations is a strong predictor of academic achievement, students should be exposed to stress management techniques to prevent consequences.

This type of study can provide information regarding changing response to stress and can help medical teachers to understand more about stress among their students and guide them to improve their academic performance. The medical faculty as well as students should be made aware of the negative consequences of stress faced during medical training and an efficient relaxation program as well as counseling services should be provided to such stressed students to enhance their academic achievements.

This study needs to be performed on larger scale along with the various other factors like gender differences, socio-economic background etc that may affect performance .This was the limitation of our study.

\section{Summary}

The present study was carried out to know the impact of examination stress on academic score in first year MBBS students.

The study revealed that, the group with elevated anxiety was associated with significantly increased serum cortisol levels and decreased in post-examination period.

In most of the students expected pattern of increased anxiety during examination and decreased anxiety scores in post-examination were observed. Compared to male students, female students showed high level of cortisol during examinations, which supported the evidence of increased stress response in females. Examination performance is affected adversely by increased stress level measured by the parameters mentioned above.

The results of this study will help to understand the pattern of response to the examination stress and enable development of strategies that will assist the students to handle the stress in a more efficient manner.

\section{References}

1. Arndt, C. B., Guly, U. M. V., McManus. Preclinical anxiety: The stress associated with a viva voce examination. Medical Education. 1986; 20: 274-80

2. Malarkey, W. B., Pearl, D. K., Demers, L. M., Kiecolt-Glaser, J. K., Flaser, R. Influence of academic stress and season on 24 hour mean concentration of acth cortisol and $\beta$-Endorphin. Psychoneuroendocrinology. 1995; 20(5): 499-508.

3. Misra, R., Mckean, M., West, S., Russo, T. Academic stress of college students: 
comparison of student and faculty perceptions. College Student Journal. 2000; June

4. Boudarene, M., Legros, J. J., TimsitBerthier, M. Study of the stress response: role of anxiety, cortisol and DHEAs. Encephale 2002 28(2): 139-46.

5. Radcliffe. C., Lester H. Perceived stress during undergraduate medical training a qualitative study. Medical Education. 2003; 37: 32-8.

6. Jenny, F. Levels and sources of stress in medical students. British Medical Journal. 1986; 292: 1177-80.

7. Saipanish, R. Stress among medical students in a Thai medical school. Med. Teach. 2003; 25(5): 502-6.

8. Lupien, S. J., Gaudreau, S., Tchiteya, B. M., Maheu, F., Sharma, S. Nair, N. P. V. et al. Stress-induced declatative memory impairment in healthy elderely subjects: Relationship to cortisol reactivity. The Journal of Clinical Endocrinology and Metabolism 1997; 82(7): 2070-5.

9. Stewart, S. M., Betson, C., Marshall, I., Wong, C. M., Lee, P. W. H., Lam, T. H. Stress and vulnerability in medical students. Medical Education. 1995; 29: 119-27.

10. Miller, P. The first year at medical school: Some findings and student perceptions. Med. Edu. 1994; 28: 5-7.

11. Singh R, Tiwari $S$ et al. Effect of examination stress on mood, performance and cortisol levels in medical students. IJPP.2012;56 (1): 48-55. 